\title{
Dyck Paths, Motzkin Paths and Traffic Jams
}

\author{
R. A. Blythe \\ Department of Physics and Astronomy, University of Manchester, Manchester, M13 \\ 9PL, England
}

\section{W. Janke}

Institut für Theoretische Physik, Universität Leipzig, Augustusplatz 10/11, 04109

Leipzig, Germany

\section{A. Johnston}

School of Mathematical and Computer Sciences, Heriot-Watt University, Riccarton, Edinburgh EH14 4AS, Scotland

\section{R. Kenna}

School of Mathematical and Information Sciences, Coventry University, Coventry, CV1 5FB, England

\begin{abstract}
It has recently been observed that the normalisation of a one-dimensional out-of-equilibrium model, the Asymmetric Exclusion Process (ASEP) with random sequential dynamics, is exactly equivalent to the partition function of a two-dimensional lattice path model of one-transit walks, or equivalently Dyck paths. This explains the applicability of the Lee-Yang theory of partition function zeros to the ASEP normalisation.

In this paper we consider the exact solution of the parallel-update ASEP, a special case of the Nagel-Schreckenberg model for traffic flow, in which the ASEP phase transitions can be intepreted as jamming transitions, and find that Lee-Yang theory still applies. We show that the parallel-update ASEP normalisation can be expressed as one of several equivalent two-dimensional lattice path problems involving weighted Dyck or Motzkin paths. We introduce the notion of thermodynamic equivalence for such paths and show that the robustness of the general form of the ASEP phase diagram under various update dynamics is a consequence of this thermodynamic equivalence.

PACS numbers: 05.40.-a, 05.70.Fh, 02.50.Ey
\end{abstract}




\section{Introduction}

It is a fact of equilibrium statistical-mechanical life that if one wishes to establish the thermodynamics of a model system it is necessary to calculate its partition function. When dealing with nonequilibrium stationary states (for example those that carry a current) it is not obvious that the object analogous to the equilibrium partition function, namely an appropriately defined normalisation of the stationary distribution, encodes the thermodynamics in a similar way.

By now the Lee-Yang zeros of such a normalisation for a number of nonequilibrium steady states have been studied in the complex plane of the microscopically irreversible transition rates that define a particular model. In particular, various one-dimensional driven diffusive systems [1, 2, reaction-diffusion processes [3, 4, 5] and an urn model for the separation of sand [6] have been investigated and, in each case, the zeros approach the real axis at the appropriate transition points (aspects of this work have been reviewed in [7]). Furthermore, the locus (i.e., impact angle) and density of the zeros near the transition point correspond to the order of the phase transition (defined in physical terms according to whether there is, e.g., phase coexistence or a diverging correlation length) in exactly the same way as for the equilibrium systems long ago considered by Lee and Yang 8, 9.

A striking feature of these various analyses is that the role of the equilibrium fugacities in the Lee-Yang approach is taken up by the nonequilibrium transition rates present in the model. The aim of this work is to explore this connection more deeply, the main result being that one can find equilibrium models with the same phase behaviour as the original nonequilibrium model, but within which the transition rates are transparently fugacities.

At a rather abstract level, this phenomenon can be understood in terms of a graph-theoretic description of the microscopic process [10, 11]. In this picture, one considers a graph in which each vertex corresponds to a microscopic configuration and directed edges to the transitions permitted in an elementary timestep. A representation of the steady-state normalisation can then be shown to coincide with the generating function of spanning in-trees on this graph [12]. Explicitly, if the microscopic transition rates (or, in a discrete-time process, transition probabilities) are drawn from the set $\left\{w_{1}, w_{2}, \ldots, w_{N}\right\}$, the normalisation $Z$ can be expressed as

$$
Z=\sum_{m_{1}, \ldots, m_{N}} n\left(m_{1}, \ldots, m_{N}\right) w_{1}^{m_{1}} w_{2}^{m_{2}} \cdots w_{N}^{m_{N}},
$$

in which $n\left(m_{1}, \ldots, m_{N}\right)$ counts the number of spanning in-trees on the graph of configurations with $m_{i}$ edges corresponding to a transition that occurs with rate $w_{i}$. Thus the rates $w_{i}$ act as fugacities that control the amount to which various classes of spanning in-tree contribute to the overall normalisation. One can see there is the possibility for different classes of trees to dominate the normalisation as the fugacities that weight the trees are varied. One may conjecture that such changes correspond to thermodynamic phase transitions in the microscopic model. 
The representation of the normalisation (11) is appealing in that it is uniquely defined for any stochastic process with a single steady state and polynomial in the variables $w_{i}$. In principle, this form can be obtained by summing the principal minors derived from the matrix of transition rates, wherein each summand corresponds to the steady-state weight of a particular configuration [10, 13. In practice, however, evaluation of the determinants involved is intractable, and in the cases where nonequilibrium steady-state distributions have been obtained via specialised methods, one typically finds polynomials that are lower in degree than (11). We believe that in these cases, the two distributions are related by a factor common to all the configurational weights which does not develop any nonanalyticities in the thermodynamic limit. In this instance, a Lee-Yang analysis gives the same results independently of the form of the normalisation studied and therefore we do not worry unduly that the normalisation is generally not uniquely defined.

A greater difficulty with expressions of the form (11) is that the relationship between fugacities and physical observables is unclear. Recently, however, Brak et al. [11] elucidated the connection for the asymmetric simple exclusion process (ASEP) with open boundaries, a model that was solved exactly and independently in [14] and [15]. There, the (reduced) normalisation of the ASEP was related to the partition function of an equilibrium surface model in which the two transition rates associated with the boundaries turn out to be fugacities conjugate to the densities of surface contacts with the horizontal axis from above and below. Thus for this model, there is an interpretation of the normalisation from which the thermodynamics can be extracted using standard equilibrium statistical mechanical techniques.

This result raises the question of whether the relationship between nonequilibrium transition rates and equilibrium fugacities extends to other nonequilibrium steady states. In this work we investigate this question by revisiting another exactly-solved variant of the ASEP, namely that with parallel lattice updates [16, 17, 18. Whereas the case with a random-sequential updating scheme, referred to above, was introduced to model the kinetics of biopolymerisation [19], the parallel version arises as a special case of the Nagel-Schreckenberg model of traffic flow [20]. From this point of view, the phase transitions in the model's steady state can be related to traffic jamming.

We begin by recalling the definition of the parallel-update ASEP along with some key results. In addition to the two boundary parameters of the random-sequential variant, there is a further quantity, $p$, which relates to the degree of parallelism in the dynamics. This gives rise to a normalisation with a considerably more complicated structure than that for the random-sequential ASEP. It is therefore appropriate to check that the Lee-Yang zeros of this normalisation correctly reproduce the known phase behaviour for the model in the complex plane of the defining transition probabilities. This being the case, we move on to establish partition functions for equilibrium surfaces or, equivalently, random walks on two different lattices that have the same thermodynamic phase behaviour as the parallel-update ASEP (and thence jamming in its guise as a traffic model). In each case the extra parameter, $p$, generalises the surfaces or walks obtained for the random-sequential ASEP in a physically meaningful 


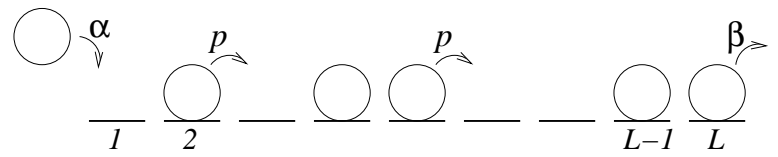

Figure 1. Dynamics of the ASEP. The arrow labels indicate the probabilities with which the corresponding transitions occur; site labels are also indicated. Note that the moves are conducted in parallel.

way. In one case, $p$ is a fugacity related to the density of horizontal segments of walks on a triangular lattice and in the other it is related to the density of peaks on the surface. In order to obtain these results we find that it is of great benefit to consider a grand-canonical ensemble in which the system size is a quantity exhibiting equilibrium fluctuations about its mean. By considering the general equilibrium thermodynamic properties of the surfaces, we are able to gain a further insight into the robustness of the phase diagram for the ASEP under different microscopic updating schemes.

\section{The Parallel-Update ASEP}

The microscopic dynamics of the asymmetric simple exclusion process with parallel dynamics are as shown schematically in Fig. 1 and defined as follows. Particles are introduced with probability $\alpha$ in each timestep at the start of a one-dimensional lattice with $L$ sites (if the first site is empty) and leave with probability $\beta$ at the other end. They can hop with probability $p$ one unit to the right if that space is empty (or remain still with probability $1-p$ ), otherwise if the space to the right is blocked they remain stationary. The update is applied to all particles simultaneously in contrast to the random sequential update scheme. Nevertheless, the latter is recovered in the limit $p \propto \mathrm{d} t \rightarrow 0$ (where $\mathrm{d} t$ is the size of each timestep) if $\alpha$ and $\beta$ are rescaled to remain proportional to $p$.

Quantities such as the current and density correlation functions for the ASEP with fully parallel dynamics $(p=1)$ were first worked out by Tilstra and Ernst [16]. Although a cluster approximation was used, the results obtained were believed to be correct in the thermodynamic limit $(L \rightarrow \infty)$. This claim was subsequently confirmed in the independent works of [17] and [18] which employed two different matrix product ansätze [14, 23] to yield exact results for general $p$. Furthermore, these calculations provide the representations of the normalisation required for the present work.

These arise by finding the steady state weights $f(\mathcal{C})$ for each configuration $\mathcal{C}$ that satisfy the stationarity condition

$$
\sum_{\mathcal{C}^{\prime} \neq \mathcal{C}}\left[f\left(\mathcal{C}^{\prime}\right) W\left(\mathcal{C}^{\prime} \rightarrow \mathcal{C}\right)-f(\mathcal{C}) W\left(\mathcal{C} \rightarrow \mathcal{C}^{\prime}\right)\right]=0
$$

in which $W\left(\mathcal{C} \rightarrow \mathcal{C}^{\prime}\right)$ is the probability of making the transition from configuration $\mathcal{C}$ to $\mathcal{C}^{\prime}$ in a single timestep. A normalisation is then obtained through

$$
Z=\sum_{\mathcal{C}} f(\mathcal{C}) .
$$


As remarked in the introduction, the weights are defined only up to an overall scale. In the calculations of [17, a reduced form of the full expression (11) was given in terms of the quantities

$a_{n, r}=\sum_{t=0}^{n-r}\left[\left(\begin{array}{c}n \\ r+t\end{array}\right)\left(\begin{array}{c}n-r-1 \\ t\end{array}\right)-\left(\begin{array}{c}n+1 \\ r+t+1\end{array}\right)\left(\begin{array}{c}n-r-2 \\ t-1\end{array}\right)\right](1-p)^{t}$

along with the conventions $\left(\begin{array}{c}X \\ 0\end{array}\right)=1$ and $\left(\begin{array}{c}X \\ -1\end{array}\right)=0$ for any integer $X$.

With these definitions the normalisation $Z_{L}$ is

$$
Z_{L}=Z_{L}^{\prime}+p Z_{L-1}^{\prime}
$$

where

$$
Z_{L}^{\prime}=\sum_{r=0}^{L} a_{L, r} \frac{(p(1-\beta) / \beta)^{r+1}-(p(1-\alpha) / \alpha)^{r+1}}{(p(1-\beta) / \beta)-(p(1-\alpha) / \alpha)} .
$$

This should be contrasted with the simpler expression for the ASEP with random sequential updates

$$
\tilde{Z}_{L}=\sum_{r=1}^{L} \frac{r(2 L-1-r) !}{L !(L-r) !} \frac{(1 / \beta)^{r+1}-(1 / \alpha)^{r+1}}{(1 / \beta)-(1 / \alpha)},
$$

in which $\alpha$ and $\beta$ are transition rates rather than probabilities.

It is also possible to define grand-canonical partition functions [17, 21, 24] by summing over system sizes with fugacity $z$ in both cases, so that $\mathcal{Z}_{p}(z)=\sum_{L} Z_{L} z^{L}$ in the parallel case and $\tilde{\mathcal{Z}}(z)=\sum_{L} \tilde{Z}_{L} z^{L}$ in the sequential one. These definitions yield

$$
\mathcal{Z}_{p}(z)=\frac{\alpha \beta(1+p z)\left[2(1-p)\left(\alpha \beta-p^{2} z\right)-\alpha \beta b^{2}(1-p z)-\alpha \beta b^{2} \sqrt{(1+p z)^{2}-4 z}\right]}{2 p^{4}(1-\beta)(1-\alpha)\left(z-z_{h d}\right)\left(z-z_{l d}\right)},
$$

where

$$
b^{2}=\frac{p}{\alpha \beta}[(1-p)-(1-\alpha)(1-\beta)]
$$

and

$$
\begin{aligned}
& z_{l d}=\frac{\alpha(p-\alpha)}{p^{2}(1-\alpha)}, \\
& z_{h d}=\frac{\beta(p-\beta)}{p^{2}(1-\beta)},
\end{aligned}
$$

in the parallel case [17] and

$$
\tilde{\mathcal{Z}}(z)=\frac{4 \alpha \beta}{(1-2 \alpha-\sqrt{1-4 z})(1-2 \beta-\sqrt{1-4 z})},
$$

in the sequential case [21, 22, 24].

The phase diagram can be extracted by considering the large $L$ behaviour of $Z_{L}$ and $\tilde{Z}_{L}$, either directly or from the asymptotics of the grand-canonical generating functions. In the parallel case using the latter approach [17] we can see that the leading singularities 


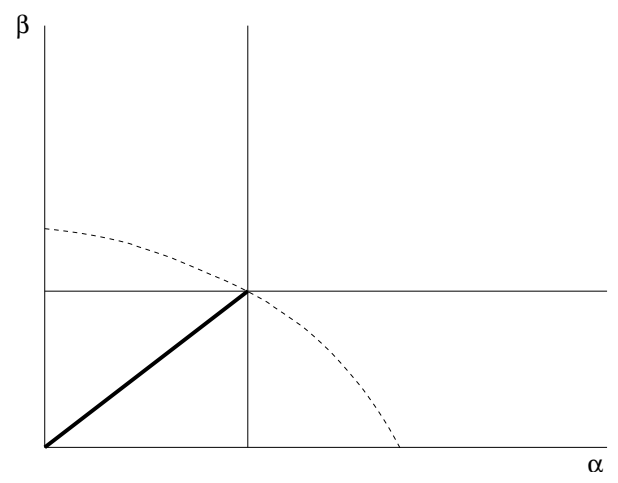

Figure 2. Phase diagram of the ASEP with parallel-update dynamics. The secondorder lines are at $\alpha, \beta=1-\sqrt{1-p}$ and the first-order line in bold $\alpha=\beta$ runs from the origin to meet these. Also indicated as a dotted line is the locus $1-p=(1-\alpha)(1-\beta)$ along which the mean field solution is exact.

in (8) come from the poles at $z_{l d}, z_{h d}$ in the low- and high-density phases respectively, or from the square root singularity,

$$
z_{m c}=\frac{2-p-2 \sqrt{1-p}}{p^{2}},
$$

in the maximal current phase.

The phase diagrams for the random sequential and parallel updates have similar topologies. That for the parallel case is shown in Fig. 2] where the main features to remark upon are the second-order transition lines at $\alpha$ or $\beta=1-\sqrt{1-p}$ and the firstorder line, depicted in bold, $\alpha=\beta<1-\sqrt{1-p}$. In addition, the mean field solution is exact along the dotted locus $1-p=(1-\alpha)(1-\beta)$. The region in which both $\alpha$ and $\beta$ are greater than $1-\sqrt{1-p}$ is a maximal current phase, that with $\alpha<1-\sqrt{1-p}$ and $\beta>\alpha$ is a low-density phase, and that with $\beta<1-\sqrt{1-p}$ and $\alpha>\beta$ is a high-density phase. There are additional transitions within these phases in which the bulk remains the same but the boundary profiles change and these occur at $\alpha$ or $\beta=1-\sqrt{1-p}$. In a similar vein the phase diagram for the random sequential updates ASEP shows a maximal current phase for $\alpha$ and $\beta>1 / 2$ separated from high- and low-density phases by second order transition lines $\alpha=1 / 2, \beta>1 / 2$ and $\beta=1 / 2, \alpha>1 / 2$. There, the high- and low-density phases are separated by a first-order line $\alpha=\beta<1 / 2$.

\section{Lee-Yang Zeros}

It has been observed that, for non-equilibrium systems, a possible equivalent of the reduced free energy might be given by

$$
f=-\lim _{L \rightarrow \infty} \frac{1}{L} Z_{L} .
$$

Since for parallel-update dynamics the singularities of (8) determine the asymptotic behaviour of the coefficients $Z_{L}$, we can see that these will simply be given by

$$
f=\ln z_{m c}, \quad \text { for } \alpha, \beta>1-\sqrt{1-p},
$$


Dyck Paths, Motzkin Paths and Traffic Jams

$$
\begin{aligned}
& f=\ln z_{l d}, \quad \text { for } \beta>\alpha, \quad \alpha<1-\sqrt{1-p}, \\
& f=\ln z_{h d}, \quad \text { for } \alpha>\beta, \quad \beta<1-\sqrt{1-p} .
\end{aligned}
$$

For random sequential updates the singularities in (12) give

$$
\begin{array}{ll}
\tilde{f}=\ln \frac{1}{4}, \quad \text { for } \alpha, \beta>1 / 2, \\
\tilde{f}=\ln \alpha(1-\alpha), \quad & \text { for } \beta>\alpha, \alpha<1 / 2, \\
\tilde{f}=\ln \beta(1-\beta), & \text { for } \alpha>\beta, \beta<1 / 2,
\end{array}
$$

where $\tilde{f}$ is similarly defined as $-\lim _{L \rightarrow \infty} \tilde{Z}_{L} / L$.

It has been suggested that for the random sequential update ASEP and other similar non-equilibrium models the Lee-Yang picture of equilibrium phase transitions [8, 9, 25] might still apply [1]. The starting point of Lee and Yang's work was the consideration of how the non-analyticity characteristic of a phase transition appear given that the partition function is a polynomial on finite lattices or graphs. The form of the reduced free energy makes it clear that any non-analyticities are associated with zeros of the partition function when the appropriate fugacities (e.g. $y=\exp (-2 h)$ for a spin model in field) are extended into the complex plane. In general, for a lattice with $L$ sites, the partition function can be written as a polynomial in the fugacity,

$$
Z_{L}=\sum_{r} D_{r} y^{r}
$$

where the degree of the polynomial is proportional to $L$. As the polynomial may be completely expressed in terms of its zeros, $y_{r}$, so too may the (reduced) finite-size free energy:

$$
f_{L}(h) \sim-\frac{1}{L} \ln \prod_{r}\left(y-y_{r}(h)\right) .
$$

In the thermodynamic limit, $L \rightarrow \infty$, the zeros move in to pinch the real axis at the transition point (or points) and the entire phase structure of the model is determined by their limiting locus and density. From (18), one has

$$
f(h) \sim-\int_{C} d y \rho(y) \ln (y-y(C))
$$

where $C$ represents some set of curves, or possibly regions, in the complex $y$-plane on which the zeros have support and $\rho(y)$ is the density of the zeros there.

The expressions for the reduced free energy for the ASEP allow a direct determination of the locus of partition function zeros. In general, the partition function zeros delineate the boundaries of different phases since they give the loci along which the free energy is singular, signalling a phase transition. In the Lee-Yang approach one considers an extension to complex parameters, so the free energy, $f$, becomes a complex quantity, $f_{l}$, which is the metastable free energy per unit volume in the various phases given by $l=1,2$. , with $\operatorname{Re} f_{l}=f$ characterising the free energy when phase $l$ is stable. The loci of zeros are then determined by demanding $\operatorname{Re} f_{i}=\operatorname{Re} f_{j}$ for adjacent phases $i, j$. Since we have $f=\ln z_{m c, h d, l d}$ for the various phases of the parallel-update 
ASEP, we can immediately see that the locus of zeros for the first-order transition will be determined by

$$
\left|z_{h d}\right|=\left|z_{l d}\right|, \quad \alpha, \beta<1-\sqrt{1-p} .
$$

Substituting in for $z_{h d}, z_{l d}$ from (10) and (11), this gives

$$
\left|\frac{\beta(p-\beta)}{p^{2}(1-\beta)}\right|=\left|\frac{\alpha(p-\alpha)}{p^{2}(1-\alpha)}\right| .
$$

Similarly, the loci of zeros for the second order transitions are determined by $\left|z_{h d}\right|=\left|z_{m c}\right|$ and $\left|z_{l d}\right|=\left|z_{m c}\right|$ or, from (10), (11) and (13)

$$
\begin{aligned}
& \left|\frac{\beta(p-\beta)}{p^{2}(1-\beta)}\right|=\left|\frac{2-p-2 \sqrt{1-p}}{p^{2}}\right| \\
& \left|\frac{\alpha(p-\alpha)}{p^{2}(1-\alpha)}\right|=\left|\frac{2-p-2 \sqrt{1-p}}{p^{2}}\right| .
\end{aligned}
$$

These formulae are analogous to those observed in the case of the first-order transition line in random sequential dynamics

$$
|\alpha(1-\alpha)|=|\beta(1-\beta)|,
$$

and for the second order lines

$$
|\gamma(1-\gamma)|=1 / 4,
$$

where $\gamma=\alpha, \beta$ depending on the region of the phase diagram.

For definiteness we now consider the zeros of the normalisation for parallelupdate dynamics given in (7) with a convenient rational value of $p=16 / 25$. This places the horizontal and vertical second order transition lines of Fig. 2 at $\alpha$ and $\beta=1-\sqrt{1-p}=2 / 5$, respectively. If we consider the former, we can choose a fixed $\beta$ value, say $\beta=3 / 5$, which will take us across this line as $\alpha$ is varied. Substituting $p=16 / 25, \beta=3 / 5$ into the second of (22) gives the analytic locus of zeros in the complex $\alpha$-plane which is plotted in Fig. 3. We have also used (6) to calculate the zeros numerically for system sizes up to $L=250$, and these are plotted in Fig. 3, showing quite good agreement with the limiting analytical results. Similarly we can take a different value of $\beta$ which crosses the first-order line, say $\beta=1 / 10$, and use (201) determine the analytic locus, which is shown in Fig. 4 along with the numerically determined zeros for a system of size $L=250$.

Referring to the discussion of the partition function zeros for the random sequential update ASEP in [2], we thus see the Lee-Yang zeros technique still captures the nature of phase transitions with parallel updates. In Fig. 3 the zeros approach the phase transition point at $\alpha=2 / 5$ at a angle of $\pi / 4$ as might be expected for a second order phase transition. In the first-order case in Fig. 4 the zeros approach the transition point at $\alpha=\beta=1 / 10$ at an angle $\pi / 2$, which is characteristic of a first-order transition.

It is also possible to look at zeros of the normalisation in the complex $p$-plane for given $\alpha$ and $\beta$. In this case one would expect to see a transition at the larger of $1-(1-\alpha)^{2}$ and $1-(1-\beta)^{2}$. The locus of zeros can be obtained by using, 


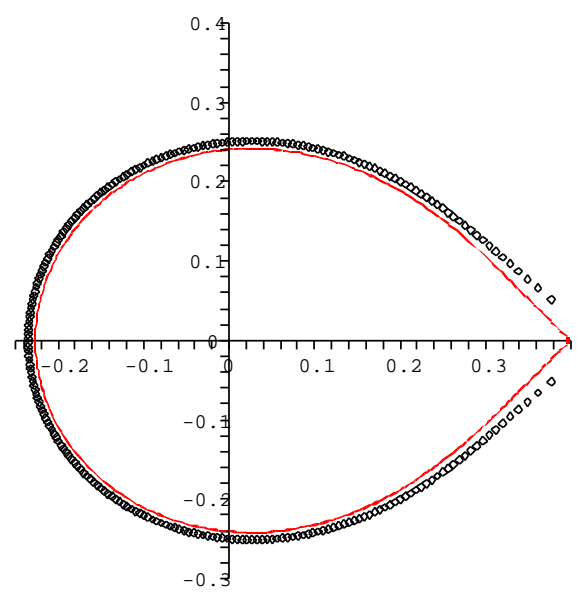

Figure 3. The locus of zeros in the complex $\alpha$-plane for the parallel-update ASEP with $p=16 / 25, \beta=3 / 5$, characteristic of a second-order transition. The diamonds show for comparison numerically determined zeros for a system of size $L=250$.

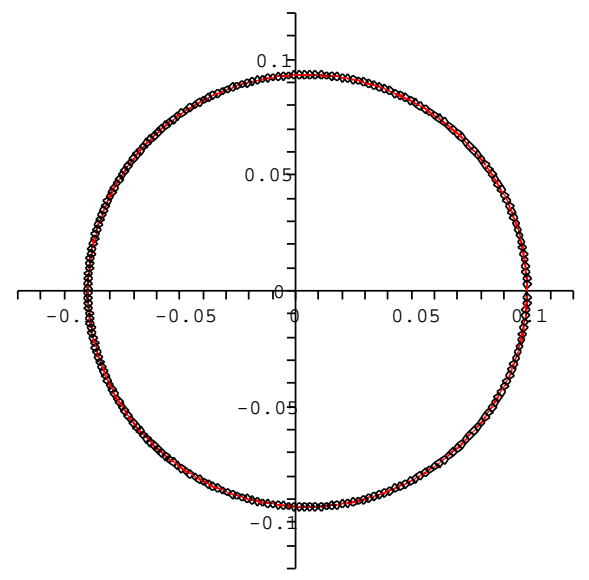

Figure 4. The locus of zeros in the complex $\alpha$-plane for the parallel-update ASEP with $p=16 / 25, \beta=1 / 10$ characteristic of a first-order transition. Again numerically obtained zeros for a system of size $L=250$ are superimposed.

e.g. $\left|\beta(p-\beta) /\left(p^{2}(1-\beta)\right)\right|=\left|(2-p-2 \sqrt{1-p}) / p^{2}\right|$ for fixed $\beta$ and complex $p$. The resulting cardioid locus is shown in Fig. 5, with the transition appearing as the cusp on the real axis. 


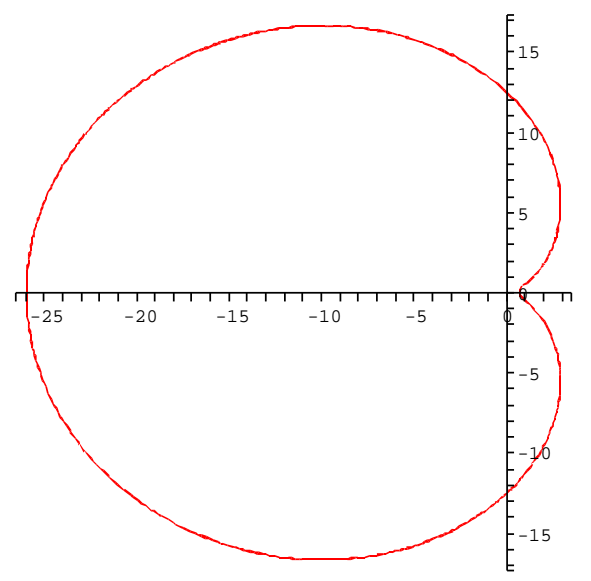

Figure 5. The locus of zeros in the complex $p$-plane for the parallel-update ASEP with $\beta=2 / 5$. The transition point is given by the cusp on the real axis at $1-(1-\beta)^{2}=16 / 25=0.64$.

\section{Thermodynamically Equivalent Equilibrium Models}

The Lee-Yang analysis of the nonequilibrium normalisation for the parallel-update ASEP in the preceding section suggests that the transition probabilities defining the model are related to equilibrium fugacities. To establish this connection concretely, we seek equilibrium models which are thermodynamically equivalent to the ASEP with parallel dynamics, which we define to occur when

(i) the extensive part of the equilibrium free energy is identical to that of the logarithm of the nonequilibrium normalisation specified by equations (44)-(6); and

(ii) the parameters $\alpha, \beta$ and $p$ are conjugate to physical densities in the equilibrium model.

A consequence of this definition is that (given the foregoing Lee-Yang analysis of the nonequilibrium normalisation) such equilibrium models will have the same thermodynamic phase behaviour. An example of a thermodynamic equivalence was recently provided for the random-sequential limit $p \rightarrow 0$ by Brak et al. [11, 27].

Our method of choice in establishing such equivalences in the more general case of parallel dynamics is to relate the grand-canonical normalisation (8) to generating functions for various types of lattice walks. In an earlier work [21] we demonstrated that in the limit $p \rightarrow 0$ this approach is technically simpler than transforming the transfer matrices that build up the lattice-path partition functions to the matrix product expressions used to solve the ASEP, which was the method used in [11, 27].

To this end we first note that (8) can be expressed as

$$
\mathcal{Z}_{p}(z)=\frac{\alpha \beta\left(1-x_{-}(z)^{2} / p\right)}{\left(\alpha-x_{-}(z)\right)\left(\beta-x_{-}(z)\right)},
$$




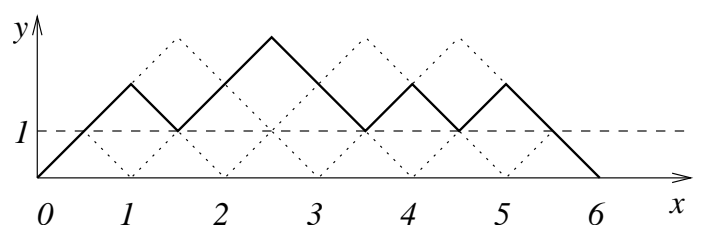

Figure 6. An excursion on the rotated square lattice. Note that between the initial and final steps, the excursion comprises sub-excursions away from the line $y=1$ and that the length of the path along the $x$-axis is equal to the number of up-down pairs.

where

$$
x_{-}(z)=\frac{p}{2}\left((1+p z)-\sqrt{(1+p z)^{2}-4 z}\right)
$$

is one of the roots of the pole terms,

$$
z=\frac{x(p-x)}{p^{2}(1-x)}
$$

appearing in the grand-canonical partition function.

Let us begin by briefly recapitulating the limit $p \rightarrow 0$ for which the equilibrium equivalence has been established [11, 21, 27], since the methods we employ will be of utility in the general case. It is clear from (26) that the function $\tilde{x}_{-}=x_{-} / p$ remains finite in the limit $p \rightarrow 0$. Therefore, to keep (25) finite we write

$$
\mathcal{Z}_{p}(z)=\frac{[\alpha / p][\beta / p]\left(1-p\left[x_{-}(z) / p\right]^{2}\right)}{\left([\alpha / p]-\left[x_{-}(z) / p\right]\right)\left([\beta / p]-\left[x_{-}(z) / p\right]\right)},
$$

which indicates the limit $p \rightarrow 0$ must be taken with $\alpha / p=\tilde{\alpha}, \beta / p=\tilde{\beta}$ as claimed in Section 2. Now note, from (27), that the function $\tilde{x}_{-}(z)$ satisfies

$$
z=\tilde{x}_{-}\left(1-\tilde{x}_{-}\right)
$$

in the limit $p \rightarrow 0$. This expression implies that $\tilde{x}_{-}$is the generating function of the number of excursions on the $45^{\circ}$ rotated square lattice, i.e. paths that touch the $x$ axis only at the start and end. The fugacity $z$ is conjugate to the length of the path, measured in terms of the number of pairs of up- and down-steps - see Fig. [6]

The generating function for excursions, $G_{E}(z)$, can be derived by observing from Fig. [6] that each comprises an initial up-step and final down-step (which together contribute a fugacity $z$ ) between which any non-negative number of excursions away from the line $y=1$ can occur. Therefore $G_{E}(z)$ can be expanded as

$$
G_{E}(z)=z\left(1+G_{E}(z)+\left[G_{E}(z)\right]^{2}+\left[G_{E}(z)\right]^{3}+\cdots\right)=\frac{z}{1-G_{E}(z)} .
$$

Comparison with (29) indicates that $\tilde{x}=G_{E}(x)$ as claimed.

To complete the path interpretation of (25) in the random-sequential limit, we recast in terms of the rescaled quantities to obtain

$$
\tilde{\mathcal{Z}}(z)=\lim _{p \rightarrow 0} \tilde{\mathcal{Z}}_{p}(z)=\frac{1}{\left(1-\tilde{x}_{-} / \tilde{\alpha}\right)\left(1-\tilde{x}_{-} / \tilde{\beta}\right)},
$$




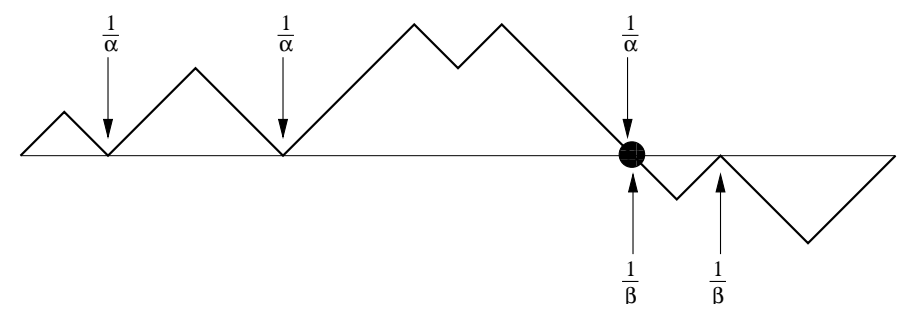

Figure 7. A one-transit walk obtained by composing two Dyck paths, one above the axis and one below. Each contact with the $x$-axis from above, apart from the start, gives a factor $1 / \alpha$ and each contact from below, apart from the end, gives a factor of $1 / \beta$.

in agreement with 21, 24]. Expansion of the denominator reveals that this expression describes an ensemble of paths with two sets of excursions, one with a fugacity $1 / \tilde{\alpha}$ per excursion (or, equivalently, return to the $x$-axis) and the other with fugacity $1 / \tilde{\beta}$. For convenience we take the first set of excursions to go above the $x$-axis and the second below thus defining a one-transit walk, a realisation of which is shown in Fig. (7) In the mathematical literature walks that are constrained to lie on or above the $x$-axis are often called Dyck paths (see e.g. [28]). A one-transit walk is then a composition of two Dyck paths.

We now use similar methods to establish equilibrium statistical mechanical interpretations for the parallel-update ASEP with general $p$.

\subsection{Walks on the triangular lattice}

Let us now consider excursions on a triangular lattice, often called Motzkin paths (such as that shown in Fig. 8) in which each horizontal step contributes a fugacity $u$ and an up-down pair $v$. The generating function $G_{T}(u, v)$ for these excursions can be derived as follows (but see also, e.g., [29]). As before, we note that each excursion comprises an extremal up-down pair (contributing a factor $v$ ) and in between there may be any combination of horizontal steps (each contributing a factor $u$ ) and excursions (contributing $G_{T}(u, v)$ ), including the null path. That is

$$
\begin{aligned}
G_{T}(u, v) & =v\left(1+\left[u+G_{T}(u, v)\right]+\left[u+G_{T}(u, v)\right]^{2}+\left[u+G_{T}(u, v)\right]^{3}+\ldots\right) \\
& =\frac{v}{1-u-G_{T}(u, v)} .
\end{aligned}
$$

Solving this equation one finds

$$
G_{T}(u, v)=\frac{1-u-\sqrt{(1-u)^{2}-4 v}}{2},
$$

in which the negative root is taken to ensure all coefficients appearing in the power-series expansion in $u$ and $v$ are positive.

A direct comparison of (33) with (26) yields an interpretation of the normalisation for the parallel-update ASEP in terms of triangular-lattice walks with a fugacity $\sqrt{z}$ for 


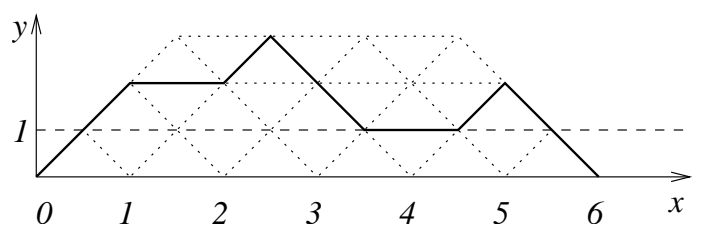

Figure 8. An excursion on the triangular square lattice. In this case both horizontal segments and sub-excursions occur on the line $y=1$. The total length of the path is the sum of the numbers of up-down pairs and horizontal segments.

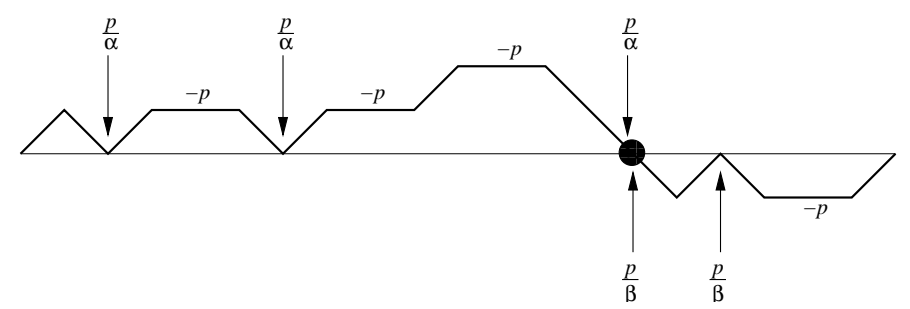

Figure 9. A one-transit walk on the triangular lattice with contact weights for the axis. The horizontal segments have a weight $-p z$ whereas up-down pairs contribute a weight $z$, so that the overall power of $z$ (not shown) is the horizontal length of the path. Meanwhile, there are contact fugacities of $p / \alpha$ from above and $p / \beta$ from below.

each up- or down-step and $-p z$ for each horizontal step. This reads

$$
\mathcal{Z}_{p}(z)=\frac{1-p G_{T}(-p z, z)^{2}}{\left(1-(p / \alpha) G_{T}(-p z, z)\right)\left(1-(p / \beta) G_{T}(-p z, z)\right)} .
$$

Note that the power of $z$ in the generating function $G_{T}(-p z, z)$ measures the horizontal length of the path as shown in Fig. 8 since each up-down pair and horizontal segment has the same length in the $x$-direction.

We remark that only the denominator is relevant in terms of the leading thermodynamic behaviour of the model. One way to see this is to note that the the factors in the numerator do not alter the location or nature of the singularities in the complex plane. More precisely, if one calculates the canonical (fixed system-size) free energy of the walk, it transpires that the numerator supplies only subextensive contributions (this point is demonstrated more explicitly in Section 5 below). Hence we have established a thermodynamic equivalence, as defined at the start of this section, between the parallel-update ASEP and the one-transit walk on the triangular lattice with fugacities $p / \alpha$ and $p / \beta$ associated with contacts from above and below, a fugacity $z$ conjugate to the path length and a negative fugacity $-p$ conjugate to the density of horizontal segments as illustrated in Fig. 9.

The origin of the negative fugacity can be understood from the discussion of the Introduction. Recall that one form of the normalisation is the generating function of spanning in-trees on the graph of configurations with edges weighted according to the elementary transition probabilities, i.e. Eq. (11). Since this model has discretetime dynamics both the probabilities $\alpha, \beta, p$ that particles move and their complements 
$1-\alpha, 1-\beta, 1-p$ that particles remain stationary appear as weights. We therefore do not require an expansion of the normalisation in powers $p$ to have positive coefficients; however, we should expect to find a series expansion in $p$ and $q=1-p$ with positive coefficients.

One can show this indeed the case for the function $G_{T}(-p z, z)$ and thence the expansion of the denominator of (34). Expanding (33) and making the change of variable $p=1-q$ one finds after some routine algebra that

$$
G_{T}(-p z, z)=z\left(1+\sum_{n \geq 1} \sum_{m=1}^{n} \frac{1}{n}\left(\begin{array}{c}
n \\
m
\end{array}\right)\left(\begin{array}{c}
n \\
m-1
\end{array}\right) z^{n} q^{m}\right),
$$

in which the coefficients of $z^{n} q^{m}$ are clearly positive. The coefficients in (35) are the Narayana numbers [30],

$$
N(n, m)=\frac{1}{n}\left(\begin{array}{c}
n \\
m
\end{array}\right)\left(\begin{array}{c}
n \\
m-1
\end{array}\right),
$$

which appear in many combinatorial contexts. Of particular note here is the fact that they are known to count Dyck paths with $2 n$ steps and $m$ peaks, so this is strongly suggestive that the parallel-update ASEP also admits an interpretation involving standard Dyck paths with weighted peaks. We shall see that this is indeed the case in the next section.

It is also possible to find a triangular lattice path interpretation of the normalisation (25) in which the quantities $p$ and $q$ appear more naturally as positive fugacities. To do this, one observes that, since $p+q=1$, (26) can be rewritten as

$$
x_{-} / p-p z=\frac{(1-p z)-\sqrt{(1-p z)^{2}-4 q z}}{2} .
$$

Now the right-hand side of this expression is equal to $G_{T}(p z, q z)$, i.e. the generating function of excursions on the triangular lattice with a fugacity $q$ associated with each up-down pair and $p$ with each horizontal step. Expressing (34) in terms of $G_{T}(p z, q z)$ yields

$$
\mathcal{Z}_{p}(z)=\frac{1+(2 p / q) G_{T}(p z, q z)-(p / q) G_{T}(p z, q z)^{2}}{\left(1-w_{a} G_{T}(p z, q z)\right)\left(1-w_{b} G_{T}(p z, q z)\right)}
$$

with

$$
\begin{aligned}
& w_{a}=\frac{p(1-\alpha)}{q \alpha}, \\
& w_{b}=\frac{p(1-\beta)}{q \beta} .
\end{aligned}
$$

As before, the terms in the numerator of (38) are thermodynamically unimportant. Therefore the phase behaviour of the parallel-update ASEP corresponds to that of a one-transit walk on the triangular lattice with contact fugacities $w_{a}$ and $w_{b}$ from above and below respectively, as shown in Fig. 10.

In both of the triangular lattice pictures one can understand the limit $p \rightarrow 0$ intuitively. In this limit, paths with horizontal segments are excluded from the partition 


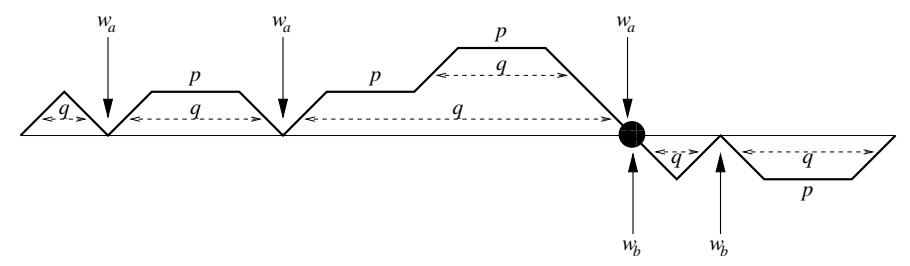

Figure 10. An alternative one-transit walk on the triangular lattice. The horizontal segments have a weight $p z$ whereas up-down pairs contribute a weight $q z$. Again the overall (omitted) power of $z$ is the horizontal length of the path. The contact fugacities from above and below are modified to $w_{a}$ and $w_{b}$ respectively.

function and one recovers the one-transit walks on the rotated square lattice as discussed above.

\subsection{Weighted-peak walks on the rotated square lattice}

Another natural extension of the one-transit walk on the rotated square lattice that arises in the case of parallel-update dynamics has $q=1-p$ conjugate to the number of peaks along the walk. By "peak" we mean here a maximum above the $x$-axis, or a minimum below.

We begin, as before, by constructing the generating function $G_{P}(z, q)$ of excursions on the rotated square lattice with the addition of a fugacity, $q$, associated with each peak (a similar derivation is given in 31]). In this instance one has between the initial up-step and final down-step (which contributes a factor $z$ ) either the null path (creating a peak with fugacity $q$ ) or any positive number of excursions in which the number of peaks is the sum of those from each excursion. Thus we obtain the expansion

$$
\begin{aligned}
G_{P}(z, q) & =z\left(q+G_{P}(z, q)+\left[G_{P}(z, q)\right]^{2}+\left[G_{P}(z, q)\right]^{3}+\cdots\right) \\
& =z\left(q+\frac{G_{P}(z, q)}{1-G_{P}(z, q)}\right) .
\end{aligned}
$$

Solving this equation and discarding the unphysical root, we find

$$
G_{P}(z, q)=\frac{(1-p z)-\sqrt{(1-p z)^{2}-4 q z}}{2},
$$

which is none other than the right-hand side of (37). Hence we have immediately a representation of the parallel-update ASEP normalisation

$$
\mathcal{Z}_{p}(z)=\frac{1+(2 p / q) G_{P}(z, q)-(p / q) G_{P}(z, q)^{2}}{\left(1-w_{a} G_{P}(z, q)\right)\left(1-w_{b} G_{P}(z, q)\right)},
$$

whose denominator describes a one-transit walk on the rotated-square lattice with each peak weighted by a fugacity $q$ and contact fugacities $w_{a}$ and $w_{b}$ associated with contacts from above and below and as given in the previous section as indicated in Fig. 11 ,

Again, the numerator of this expression is thermodynamically unimportant and we see that the limit $p \rightarrow 0$ removes the weights associated with the peaks. This 


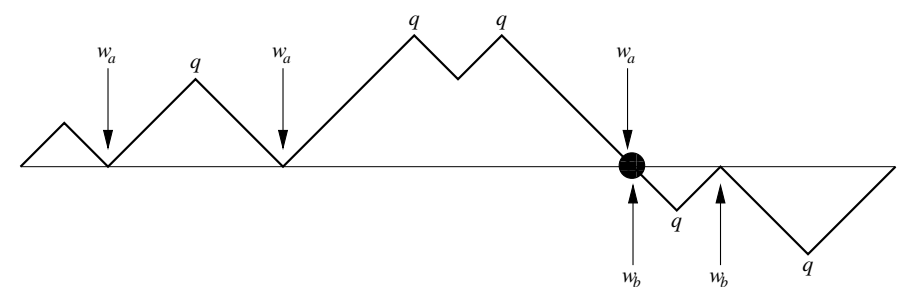

Figure 11. A weighted-peak one-transit walk on the rotated square lattice. Here there is an additional weight $q$ associated with each maximum above the $x$-axis and minimum below. The contact fugacities from above and below are the same as in Fig. [10]

corresponds, as it should, to the one-transit walks described at the start of this section in the context of random-sequential dynamics.

\subsection{Other update dynamics}

Other update dynamics for the ASEP have been extensively investigated, including sublattice parallel dynamics, and backward and forward ordered sequential dynamics [33, 34]. It was observed in [17, 18] that there was a close relation between sublattice parallel dynamics, backward and forward ordered sequential dynamics and the (totally) parallel dynamics we have discussed so far. For all these the rules for making a hop are the same, but in the sublattice parallel case all site pairs $i, i+1$ with even $i$ are updated on even time steps and all pairs with odd $i$ on odd time steps, whereas in ordered sequential updates the sites are updated one at a time starting at the left hand side and proceeding to the right (forward ordered) or right to left (backward ordered). The canonical normalisation for the sublattice parallel $(s p)$, backward ordered sequential (bos) and forward ordered sequential dynamics (fos) is given by the $Z_{L}$ of (15), which in turn gives the grand canonical normalisation $\mathcal{Z}_{s p}$, bos, fos

$$
\mathcal{Z}_{s p, b o s, f o s}(z)=\frac{\alpha \beta\left[2(1-p)\left(\alpha \beta-p^{2} z\right)-\alpha \beta b^{2}(1-p z)-\alpha \beta b^{2} \sqrt{(1+p z)^{2}-4 z}\right]}{2 p^{4}(1-\beta)(1-\alpha)\left(z-z_{h d}\right)\left(z-z_{l d}\right)},
$$

for all three updates [17.

We can again rewrite (45) in a form which is recognizably that of a generating function for one-transit walks. Using $x_{-}(z)=\frac{p}{2}\left((1+p z)-\sqrt{(1+p z)^{2}-4 z}\right)$ we have

$$
\mathcal{Z}_{s p, b o s, f o s}(z)=\frac{\alpha \beta\left(1-x_{-}(z)\right)}{\left(\alpha-x_{-}(z)\right)\left(\beta-x_{-}(z)\right)},
$$

which should be compared to the expression for parallel updates in (25). With (46) at hand any of the walk representations previously employed may be used to recast the grand-canonical normalisation in the form of a one-transit walk generating function. For instance, using the triangular lattice generating function one can write

$$
\mathcal{Z}_{s p, \text { bos, fos }}(z)=\frac{\left(1-p G_{T}(-p z, z)\right)}{\left(1-(p / \alpha) G_{T}(-p z, z)\right)\left(1-(p / \beta) G_{T}(-p z, z)\right)} .
$$


Since the singularities in (47) arise from the square root in $G_{T}(-p z, z)$ and the poles in the denominator, which are identical to the parallel case, we find the same phase diagram.

\section{Thermodynamics of One-Transit Walks}

To finish we briefly investigate the thermodynamics of one-transit walks. It is, in fact, possible to derive qualitatively the phase diagram with only a little knowledge of the detailed structure of the walks. This provides a picture within which one can understand clearly the robustness of the phase diagram of the ASEP under a variety of updating schemes 32, 33, 34.

Consider a walk of length $L$ that comprises $n_{a}$ excursions above and $n_{b}$ excursions below the $x$-axis. For added generality we associate with each walk a number, $m$, that counts some property that depends on the shape of the walk, but not on whether a particular excursion lies above or below the axis. The number of peaks is such a property. If there is an energy $\epsilon_{a}\left(\epsilon_{b}\right)$ associated with each of the $n_{a}\left(n_{b}\right)$ contacts from above (below) and an energy $\epsilon^{\prime}$ with the property counted by $m$, the free energy of the walk is

$$
F=n_{a} \epsilon_{a}+n_{b} \epsilon_{b}+m \epsilon^{\prime}-S\left(L, n_{a}, n_{b}, m\right),
$$

in which $S\left(L, n_{a}, n_{b}, m\right)$ is the entropy (logarithm of the number of realisations) of paths with fixed $n_{a}, n_{b}$ and $m$.

The consequence of $m$ being independent of whether an excursion lies above or below the axis is that

$$
S\left(L, n_{a}, n_{b}, m\right)=\ln \Omega\left(L, n_{a}+n_{b}, m\right),
$$

where $\Omega(L, n, m)$ is the number of walks of length $L$ comprising $n$ excursions and fixed $m$. It then follows that if (48) can be minimised with respect to $n_{a}, n_{b}$ and $m$ in such a way that $n=n_{a}+n_{b}$ is nonzero, this minimum is achieved by all the excursions being on the side of the $x$-axis for which the contact energy is smaller. That is,

$$
F=n \min \left\{\epsilon_{a}, \epsilon_{b}\right\}+m \epsilon^{\prime}-\ln \Omega(L, n, m) .
$$

If $\epsilon_{a}=\epsilon_{b}$, the free energy is neutral to the flipping of an excursion, and so along this coexistence line, the crossing point will wander along the $x$-axis. This is suggestive of a first-order phase transition.

It is intuitively clear that the number of possible walks increases as the number of contacts decreases. This implies that once the smaller contact energy has increased above some critical value, the entropy gained from avoiding the axis (i.e. becoming desorbed) is not offset by any energy saving in contacting the axis. Furthermore, since the free energy depends only on the smaller of contact energies, the critical value must

be independent of the larger contact energy. The effect of $\epsilon^{\prime}$ will be to favour a particular shape of path, and thus we would expect this to affect the critical contact energy at which the path becomes desorbed. These considerations give rise to a phase diagram 


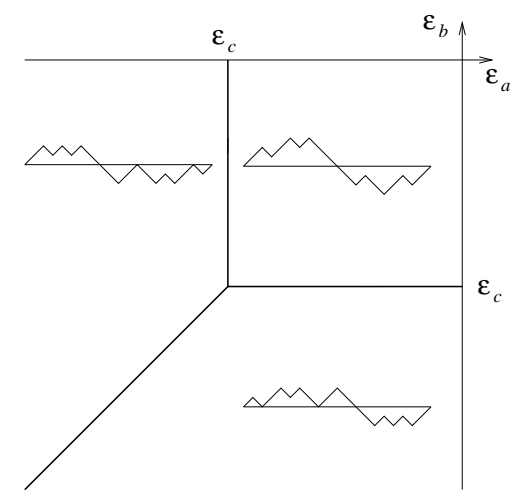

Figure 12. Generic phase diagram of a one-transit walk. The density of contacts is nonzero on the side of the axis for which the contact energy $\epsilon_{a, b}$ is lower. The (negative) critical energy $\epsilon_{c}$ at which the entropy of the walk causes it to desorb from the axis depends on $\epsilon^{\prime}$ and how it is coupled to the shape of the path.

with the topology shown in Fig. 12 which is expected be common to a large class of one-transit walks.

For concreteness, let us now consider the specific case of the peak-weighted walk illustrated in Fig. 11] which has $m$ as the number of peaks, $\epsilon_{a, b}=-\ln w_{a, b}$ and $\epsilon^{\prime}=-\ln q$. The number of walks of length $L$ comprising $n$ excursions and $m$ peaks is the coefficient of $z^{L} q^{m}$ in $\left[G_{P}(z, q)\right]^{n}$. This quantity can be obtained readily by applying the Lagrange inversion formula to the functional relation (42) for $G_{P}$ [31, 35]. This reveals that for these walks, $\Omega(L, n, m)$ is the coefficient of $u^{L-n} q^{m}$ in

$$
\frac{n}{L}\left(q+\frac{u}{1-u}\right)^{L},
$$

from which one quickly finds

$$
\Omega(L, n, m)= \begin{cases}\frac{n}{L}\left(\begin{array}{c}
L \\
m
\end{array}\right)\left(\begin{array}{c}
L-n-1 \\
m-n
\end{array}\right) & 0<n \leq m<L \\
1 & n=m=L \\
0 & \text { otherwise. }\end{cases}
$$

In the thermodynamic limit, $L \rightarrow \infty$, one finds the extensive part of the entropy to be

$$
\begin{aligned}
s(\rho, \sigma) & =\lim _{L \rightarrow \infty} \frac{1}{L} \ln \Omega(L, \rho L, \sigma L) \\
& =-[\sigma \ln \sigma+2(1-\sigma) \ln (1-\sigma)+(\sigma-\rho) \ln (\sigma-\rho)-(1-\rho) \ln (1-\rho)],
\end{aligned}
$$

in which $\rho=n / L$ and $\sigma=m / L$ are the thermodynamic densities of contacts and peaks. Minimising the free energy per unit length

$$
f=\rho \min \left\{\epsilon_{a}, \epsilon_{b}\right\}+\sigma \epsilon^{\prime}-s(\rho, \sigma),
$$

with respect to these densities one finds their equilibrium values are

$$
\begin{aligned}
\rho^{*} & =\frac{q(w-1)^{2}-1}{(w-1)(q[w-1]+1)}, \\
\sigma^{*} & =\frac{q(w-1)}{q(w-1)+1},
\end{aligned}
$$


for those values of $q$ and $w=\max \left\{w_{a}, w_{b}\right\}$ where the physical requirement $0 \leq \rho^{*} \leq$ $\sigma^{*} \leq 1$ holds. One can check that the inequality $\rho^{*} \leq \sigma^{*} \leq 1$ is automatically true whenever $\rho^{*} \geq 0$ is satisfied. The latter condition, however, is violated when $w_{c}<1+q^{-1 / 2}$. Then the free energy is minimised at some point on the boundary of the physical part of the $(\rho, \sigma)$-plane. It is easily verified that this point has $\rho^{*}=0$ (i.e. total desorption of the path) and a peak density

$$
\sigma^{*}=\frac{q^{1 / 2}}{1+q^{1 / 2}} .
$$

One then finds that in all phases the free energy takes the form

$$
f=2 \ln \left(1-\sigma^{*}\right)-\ln \left(1-\rho^{*}\right) .
$$

Using these results together with the definitions (39) and (40), one recovers the expressions for the ASEP free energy given in Section [3.

This provides explicit confirmation that the peak-weighted one-transit walk and the parallel-update ASEP are thermodynamically equivalent, despite the fact the grandcanonical partition function of the latter does not agree exactly with that for the ASEP (44) which has additional factors in its numerator. The reason for the equivalence is that these factors describe a small, fixed number of additional excursions which contribute only to the subextensive part of the entropy, and hence become irrelevant in the thermodynamic limit.

\section{Conclusions}

The Lee-Yang theory of partition function zeros has been shown to apply to the ASEP with parallel-update dynamics, correctly pinpointing the first- and second-order transition lines in the model in the complex plane of transition probabilities. This fact suggests that there is an interpretation of these transition rates as equilibrium fugacities. Indeed, we were able to make the correspondence concrete by mapping the normalisation of the ASEP onto partition functions for equilibrium path problems. We found that for parallel updates there were several possible path transcriptions, including two types of adsorbing paths on a triangular lattice and a class of peak-weighted paths on the rotated square lattice. In all cases the appropriate scaling limit recovered the one-transit walk associated with the random sequential update ASEP.

The benefit of finding such mappings is twofold. First one sees quite clearly why a Lee-Yang analysis of a nonequilibrium normalisation should be appropriate in the complex plane of transition rates or probabilities: under the mapping to an equilibrium model, one finds that they are fugacities. Secondly, standard techniques from equilibrium statistical mechanics can be used to learn about the thermodynamics of nonequilibrium models. For example, we showed that the structure of the phase diagram for one-transit walks is essentially unchanged when an additional fugacity associated with the shape of the excursions is introduced. In turn, this provides an explanation as to why parallelising the dynamics of the ASEP does not unduly change 
its phase behaviour. This phenomenon can also be understood in terms of an extremal current principle for driven diffusive processes [34, 36]. This is suggestive that that the equilibrium walk picture explored in the present work is applicable to a wider range of processes to those studied so far. Conversely, other nonequilibrium systems are known to be equivalent to equilibrium models (such as the "raise and peel" interface model [37]) so one might expect Lee-Yang theory to apply to these also. Meanwhile, we note that the path equivalences have also been utilised to calculate density fluctuations in the ASEP through a suitable interpretation of the corresponding matrix-product expressions [38].

In this work, we focussed on equilibrium models that are thermodynamically equivalent to the ASEP with parallel-update dynamics. At the start of Section 4 we defined this equivalence to exist when the extensive parts of the free energies (defined for the nonequilibrium case as the logarithm of the normalisation) coincide for the two models. In practice, this meant that the equilibrium surface models we described had partition functions that differed from the normalisation of the ASEP. This is reflected in, e.g., nontrivial numerator factors in the grand-canonical normalisations (34), (38) and (44). These factors did not concern us here, since we were interested only in the thermodynamic phase behaviour of the models: if one wishes to consider finite-sized systems, one should take care to include them. Nevertheless, it turns out that even for finite-sized systems, the loci of zeros of the normalisation obtained from an "artificial" grand-canonical generating function constructed from the denominator alone are similar to those obtained using the full expressions.

Thus far, our analyses (and related calculations in [11, 38] ) have relied on the prior existence of solutions for the nonequilibrium steady states under consideration. This is, of course, an unsatisfactory state of affairs, and ideally we would like to be able to establish the path equivalences directly from the definition of the microscopic process. We suggest, admittedly speculatively, that the extremely compact forms of the grand-canonical normalisations for the ASEP suggest that such an enterprise may be possible (though no small challenge). In particular, such a study might yield a new way to determine nonequilibrium steady-state distributions. As a first step towards this goal, it would be of interest to investigate other nonequilibrium states for which thermodynamically equivalent equilibrium models have not yet been derived. Chief among these is the partially asymmetric exclusion process (PASEP) [39, 40] in which particles may hop to the left as well as to the right across the lattice. When the left and right hop rates are equal, there is an additional transition to a phase in which there is a zero current in the thermodynamic limit. If one could understand this transition from an equilibrium statistical mechanical point of view, it would give further weight to the growing evidence that phase transitions in equilibrium and nonequilibrium steady states are two sides of same coin. 
Dyck Paths, Motzkin Paths and Traffic Jams

\section{Acknowledgements}

WJ and DAJ were partially supported by EC network grant HPRN-CT-1999-00161. RAB was supported by an EPSRC Fellowship under grant $G R / R 44768$. We thank Martin Evans and Gunter Schütz for helpful comments.

[1] P. F. Arndt, Phys. Rev. Lett. 84, 814 (2000).

[2] R. A. Blythe and M. R. Evans, Phys. Rev. Lett. 89, 080601 (2002).

[3] S. M. Dammer, S. R. Dahmen and H. Hinrichsen, J. Phys. A: Math. Gen. 35, 4527 (2002).

[4] F. H. Jafarpour J. Phys. A: Math. Gen. 36, 7497 (2003).

[5] F. H. Jafarpour, J. Stat. Phys. 113, 269 (2003).

[6] I. Bena, F. Coppex, M. Droz and A. Lipowski, Phys. Rev. Lett. 91, 160602 (2003).

[7] R. A. Blythe and M. R. Evans, Braz. J. Phys. 33, 464 (2003).

[8] C. N. Yang and T. D. Lee, Phys. Rev. 87, 404 (1952).

[9] T. D. Lee and C. N. Yang, Phys. Rev. 87, 410 (1952).

[10] R. A. Blythe, Nonequilibrium Phase Transitions and Dynamical Scaling Regimes, Ph.D. thesis, University of Edinburgh (2001).

[11] R. Brak, J. de Gier and V. Rittenberg, J. Phys. A: Math. Gen. 37, 4303 (2004).

[12] F. Harary, Graph Theory (Addison-Wesley, Reading, Mass., 1969).

[13] R. A. Blythe and M. R. Evans, Physica A 313, 110 (2002).

[14] B. Derrida, M. R. Evans, V. Hakim and V. Pasquier, J. Phys. A: Math. Gen. 26, 1493 (1993).

[15] G. Schütz and E. Domany, J. Stat. Phys. 72, 277 (1993).

[16] L. G. Tilstra and M. H. Ernst, J. Phys. A: Math. Gen. 31, 5033 (1998).

[17] M. R. Evans, N. Rajewsky and E. R Speer, J. Stat. Phys. 95, 45 (1999).

[18] J. de Gier and B. Nienhuis, Phys. Rev. E 59, 4899 (1999).

[19] C. T. MacDonald, J. H. Gibbs and A. C. Pipkin, Biopolymers 6, 1 (1968).

[20] K. Nagel and M. Schreckenberg, J. Phys. I France 2, 2221 (1992).

[21] R. A. Blythe, W. Janke, D. A. Johnston and R. Kenna, JSTAT P06001, (2004).

[22] O. J. Heilmann, Exact solution of a $1 D$ asymmetric exclusion model with variable cluster size, to appear in J. Stat. Phys. (2004).

[23] K. Krebs and S. Sandow, J. Phys. A: Math. Gen. 30, 3165 (1997).

[24] M. Depken, Models of Non-Equilibrium Systems, D.Phil. thesis, University of Oxford (2003).

[25] M. E. Fisher, Lectures in Theoretical Physics, vol. 7C (University of Colorado Press, Boulder, 1965).

[26] H. Hinrichsen, Adv. Phys. 49, 815 (2000).

[27] R. Brak and J. Essam, J. Phys. A: Math. Gen. 37, 4183 (2004).

[28] E. J. Janse van Rensburg, The Statistical Mechanics of Interacting Walks, Polygons, Animals and Vesicles (Oxford University Press, Oxford, 2000).

[29] R. Brak, A. C. Oppenheim and A. L. Owczarek, Int. J. Mod. Phys. B 16, 1269 (2002).

[30] T. V. Narayana, Sankhya 21, 91 (1959).

[31] E. Deutsch, Discrete Math. 204, 167 (1999).

[32] N. Rajewsky, L. Santen, A. Schadschneider and M. Schreckenberg, J. Stat. Phys. 92, 151 (1998).

[33] A.B. Kolomeisky, G.M. Schütz, E.B. Kolomeisky and J.P. Straley, J. Phys. A: Math. Gen. 31, 6911 (1998).

[34] V. Popkov and G.M Schütz, Europhys. Lett. 48, 257 (1999).

[35] H. S. Wilf, Generatingfunctionology (Academic Press, San Diego, 1994).

[36] J. Krug, Phys. Rev. Lett. 67, 1882 (1991).

[37] J. de Gier and B. Nienhuis, J. Stat. Phys. 114, 1 (2004).

[38] B. Derrida, C. Enaud and J. L. Lebowitz, J. Stat. Phys. 115, 365 (2004). 
[39] T. Sasamoto, J. Phys. A: Math. Gen. 32, 7109 (1999).

[40] R. A. Blythe, M. R. Evans, F. Colaiori and F. H. L. Essler, J. Phys. A: Math. Gen. 33, 2313 (2000). 